\title{
Think Globally, Search Locally *
}

\author{
Kamen Yotov, Keshav Pingali, Paul Stodghill, \\ \{kyotov, pingali, stodghil\}@cs. cornell. edu \\ Department of Computer Science, \\ Cornell University, \\ Ithaca, NY 14853.
}

November 4, 2004

\begin{abstract}
A key step in program optimization is the determination of optimal values for code optimization parameters such as cache tile sizes and loop unrolling factors. One approach, which is implemented in most compilers, is to use analytical models to determine these values. The other approach, used in library generators like ATLAS, is to perform a global search over the space of parameter values by generating different versions of the code and executing them on the actual machine to find the parameter values that give the best performance.

Neither approach is suitable for use in general-purpose compilers that must generate high quality code for large programs running on complex architectures. Model-driven optimization may incur a performance penalty of $10-20 \%$ even for a relatively simple code like matrix multiplication, as was shown recently by Yotov et al. On the other hand, global search is not tractable for optimizing large programs for complex architectures because the optimization space is too large. To address this problem, some researchers are exploring more sophisticated search algorithms such as the simplex method, but it remains to be seen if these methods are successful in reducing search time without compromising on the quality of the solution.

In this paper, we advocate a different methodology for generating high-performance code without increasing search time dramatically. Our methodology has three components: (i) modeling, (ii) local search, and (iii) model refinement. We use analytical models to estimate optimal values for transformation parameters. Since it is impossible to build tractable analytical models that capture all the features of
\end{abstract}

*This work was supported by NSF grants ACI-9870687, EIA-9972853, ACI-0085969, ACI-0090217, ACI-0103723, and ACI012140 . 
complex architectures, we advocate improving these estimates by using a local search in the neighborhood of the model-predicted values. Finally, if the performance gap between handwritten code and generated code is substantial on some architecture, we advocate model refinement.

To demonstrate this methodology, we built a modified ATLAS system that used a simple analytical model and local search, and showed that on most architectures, the performance of the code produced by this system was comparable to that of code produced by the original ATLAS system using global search. However, on x86 architectures, the gap in performance was substantial, and could not be bridged by local search alone. We argue that the problem is that the model assumed aggressive operation scheduling to mask instruction latencies, but such scheduling can actually be harmful on x86 architectures, a somewhat surprising fact that does not appear to be known widely. To address this problem, we use model refinement to generate a more sophisticated model that, when combined with local search, enables the production of high-quality code on both RISC and CISC architectures.

\section{Introduction}

Although the compiler community has invested a lot of effort in inventing program optimization strategies for producing efficient code from high-level programs $[1,2,3,4,8,9,11,14]$, the quality of code produced by current compilers can be quite poor [19]. To improve the state of the art of compilers, it is necessary to study codes for which highly tuned implementations are available; by comparing these implementations with compiler-generated code, we can understand what compilers are doing wrong. Benchmark suites like the SPEC and Perfect benchmarks are not suitable in this context since there are no highly tuned implementations of these codes to serve as the gold standard for comparisons.

Fortunately, highly tuned implementations do exist in the domain of numerical linear algebra. The key routines in this domain are the Basic Linear Algebra Subroutines (BLAS), of which matrix multiplication is the most important one. Recently, Dongarra and his co-workers have implemented a portable BLAS generator called ATLAS [17], which is publicly available. Experiments show that the code produced by ATLAS performs nearly as well as the native BLAS on many machines, so the code generated by ATLAS is an excellent object of study for researchers interested in improving the state of the art of current compilers.

Why does ATLAS produce code that performs so much better than the code produced by current compilers? Most compilers use simple architectural models to determine whether an optimization is useful, and to estimate values for parameters associated with that optimization. When tiling a loop for example, a compiler may estimate the tile size using only the size of the data cache, ignoring its associativity and line size. In contrast, a library generator like ATLAS uses empirical optimization; it produces multiple versions of the tiled loop with different tile sizes, executes all of them on the actual machine, and selects the tile 
size that gave the best performance. It is commonly believed that the shortcomings of current compilers arise because the models they use are too simplistic to permit accurate estimation of optimal values for code optimization parameters.

However, recent studies by Yotov et al [19] have cast doubt on this belief. To compare empirical optimization with model-based optimization, they built a modified version of ATLAS in which search was replaced with a module that used simple analytical models to estimate values for optimization parameters. This model is described briefly in Section 3. Somewhat surprisingly, their experiments show that on the three architectures they considered, the performance of code produced by the model-driven version of ATLAS was within $10 \%$ to $20 \%$ of the performance of code produced by ATLAS using global search. Furthermore, the time required by the model-driven version was negligible compared to the time required to perform global search. These results suggest that the use of analytical models in compilers need not come in the way of generating relatively high-quality code.

In this paper, we address two problems in using model-driven optimization in the context of generalpurpose compilers.

The first problem is the performance gap between code produced by library generators and code produced by using model-driven optimization. Although a compiler that quickly generates code that performs within $10 \%$ to $20 \%$ of highly tuned code is adequate in most situations, this performance penalty may be unacceptable for critical applications that will be run many times. On the other hand, global search as performed by ATLAS does not scale well to large programs or to complex architectures, so it cannot be used in general-purpose compilers. Dongarra and co-workers are exploring faster search algorithms like the simplex method [5], but it is not clear that these algorithms alone are adequate.

The second problem is performance portability. It may seem that the use of global search ensures that library generators will work "out of the box" on new architectures, whereas model-driven optimization may fail if the model is a poor abstraction of the new architecture. However, global search is not a panacea. In particular, if the code generator does not exploit aspects of an architecture that are key to performance, the resulting code may be poor regardless of how exhaustive the search for optimal parameter values is. The methodology used in the ATLAS system to adapt to new architectures for which search alone is not sufficient is to include a collection of user-contributed hand-tuned kernels in the distribution; during the search process, the performance of these codes is evaluated, and if one of them performs better than the code generated by the code generator, it is used to produce the library. This methodology cannot be used for model-driven optimization because it runs counter to the spirit of using models to optimize programs.

The approach that we advocate in this paper to address both problems is to use a combination of model refinement and local search. To close the performance gap with code produced by empirical optimization, 
we advocate using local search in the neighborhood of the parameter values produced by using the model. Of course local search alone may not be adequate if the model is not a good abstraction of the architecture. In that case, we advocate using model refinement in the same spirit as ATLAS incorporates user-contributed code - we study the new architecture and refine the model as needed. Note that like the production of usercontributed code, model refinement must be done manually. Intuitively, in our approach, small performance problems are tackled using local search, while large performance problems are tackled using model refinement.

The experiments reported in this paper show that the combination of model refinement and local search is effective in closing performance gaps between the model-generated code and the code generated by global search, while keeping code generation time small. However, it is important to realize that reducing library generation time is not the primary focus of our work; rather, our goal is to find optimization strategies for generating very high-performance code that can be used in general-purpose compilers because they scale to large programs and complex architectures.

The rest of this paper is organized as follows. In Section 2, we describe (i) the optimization parameters used in the ATLAS system, and (ii) the global search process used by ATLAS to find optimal values for these parameters. In Section 3, we briefly describe the model of Yotov et al [19] for computing values for transformation parameters. In Section 3.2, we discuss experimental results on a number of machines, that reveal the potential for improvements in the model (and in ATLAS). In Section 4, we describe how model refinement and local search can be used to tackle these problems in the context of model-driven optimization. In Section 5, we present experimental results for the same machines as before, showing that this methodology addresses the performance problems identified earlier. In some cases, we obtain better code than is produced by the ATLAS code generator. We conclude in Section 6 with a discussion of future work.

\section{Overview of ATLAS}

Figure 1 is a block diagram of the ATLAS system. There are two main modules: Code Generator, and Empirical Search Engine.

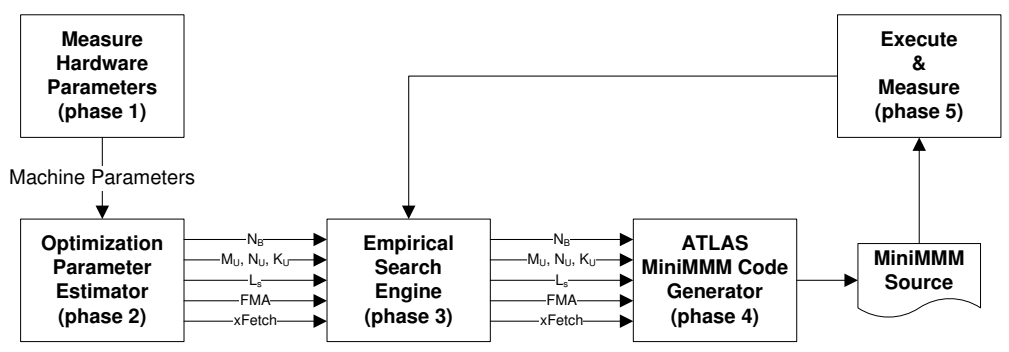

Figure 1: Empirical Optimization Architecture 
1. Code Generator: For the purpose of this paper, this module generates an optimized matrix-multiplication routine, given certain optimization parameters as input. These optimization parameters are described in more detail in Section 2.1; intuitively, they tell the code generator what tile size to use $\left(N_{B}\right)$, how much to unroll certain loops $\left(M_{U}, N_{U}, K_{U}\right)$, etc.

2. Empirical Search Engine: To determine optimal values for these code optimization parameters, ATLAS employs a global search over the space of these parameters, performed under the direction of the Empirical Search Engine. This module enumerates each point in the parameter search space, and passes it on to the code generator, which produces the appropriate matrix-multiplication code (shown as mini-MMM code in Figure 1). This code is run on the actual machine, and its performance is recorded. Once the search is complete, the parameter values that give the best performance are used to generate the library. Section 2.2 describes this search in more detail.

To finish the global search in reasonable time, it is necessary to bound the search space. When ATLAS installs itself on a machine, it runs a set of micro-benchmarks to measure a set of hardware parameters such as the L1 data cache capacity [7], the number of registers, etc. These hardware parameters are used by the Optimization Parameter Estimator module in Figure 1 to bound the search space for the optimization parameters. For example, the capacity of the L1 data cache is used to bound the search for cache tile size.

\subsection{Code optimization parameters}

To explain the role of the code optimization parameters, we use the framework of restructuring compilers to describe the code generated by ATLAS (it is important to keep in mind that ATLAS is not a general-purpose restructuring compiler). We concentrate on matrix multiplication (MMM), which is the key routine in the BLAS. Naïve MMM code is shown in Figure 2.

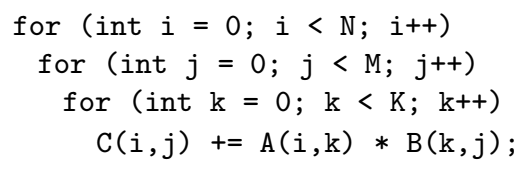

Figure 2: Naïve MMM Code

\subsubsection{Memory Hierarchy Optimizations}

The code shown in Figure 2 can be optimized by tiling for the L1 data cache and registers.

- Optimization for the L1 data cache: 
To improve locality, ATLAS implements an MMM as a sequence of mini-MMMs, where each miniMMM multiplies sub-matrices of size $N_{B} \times N_{B} . N_{B}$ is an optimization parameter whose value must be chosen so that the working set of the mini-MMM fits in the L1 cache.

In the terminology of restructuring compilers, the triply-nested loop of Figure 2 is tiled with tiles of size $N_{B} \times N_{B} \times N_{B}$, producing an outer and an inner loop nest. For the outer loop nest, code for both the JIK and IJK loop orders are implemented. When the MMM library routine is called, it uses the shapes of the input arrays to decide which version to invoke. For the inner loop nest, only the JIK loop order is used, with $\left(j^{\prime}, i^{\prime}, k^{\prime}\right)$ as control variables. This inner loop nest multiplies sub-matrices of size $N_{B} \times N_{B}$, and we call this computation a mini-MMM.

- Optimization for the register file: ATLAS converts each mini-MMM into a sequence of micro-MMMs, where each micro-MMM multiplies an $M_{U} \times 1$ sub-matrix of A with a $1 \times N_{U}$ sub-matrix of B and accumulates the result into an $M_{U} \times N_{U}$ sub-matrix of C. $M_{U}$ and $N_{U}$ are optimization parameters that must be chosen so that a micro-MMM can be executed out of the floating-point registers. For this to happen, it is necessary that $M_{U}+N_{U}+M_{U} \times N_{U} \leq N_{R}$, where $N_{R}$ is the number of floating-point registers.

In terms of restructuring compiler terminology, the $\left(j^{\prime}, i^{\prime}, k^{\prime}\right)$ loops of the mini-MMM from the previous step are tiled with tiles of size $N_{U} \times M_{U} \times K_{U}$, producing an extra inner loop nest. The JIK loop order is chosen for the outer loop nest after tiling, and the KJI loop order for the inner loop nest.

The resulting code after the two tiling steps is shown in Figure 3. To keep this code simple, we have assumed that all step sizes in these loops divide the appropriate loop bounds exactly (so $N_{B}$ divides $M, N$, and $K$, etc.). In reality, code should also be generated to handle the fractional tiles at the boundaries of the three arrays; we omit this clean-up code to avoid complicating the description. Figure 4 is a pictorial view of a mini-MMM computation within which a micro-MMM is shown using shaded rectangles.

To perform register allocation for the array variables referenced in the micro-MMM code, the microMMM loop nest $\left(j^{\prime \prime}, i^{\prime \prime}\right)$ in Figure 3 is fully unrolled, producing $M_{U} \times N_{U}$ multiply-add statements in the body of the middle loop nest. In the unrolled loop body, each array element is accessed several times. To enable register allocation of these array elements, ATLAS introduces a scalar temporary for each element of $\mathrm{A}, \mathrm{B}$, and $\mathrm{C}$ that is referenced in the unrolled micro-MMM code, and replaces array references in the unrolled micro-MMM code with references to these scalars. Appropriate assignment statements are introduced to initialize the scalars corresponding to A and B elements. In addition, assignment statements are introduced 


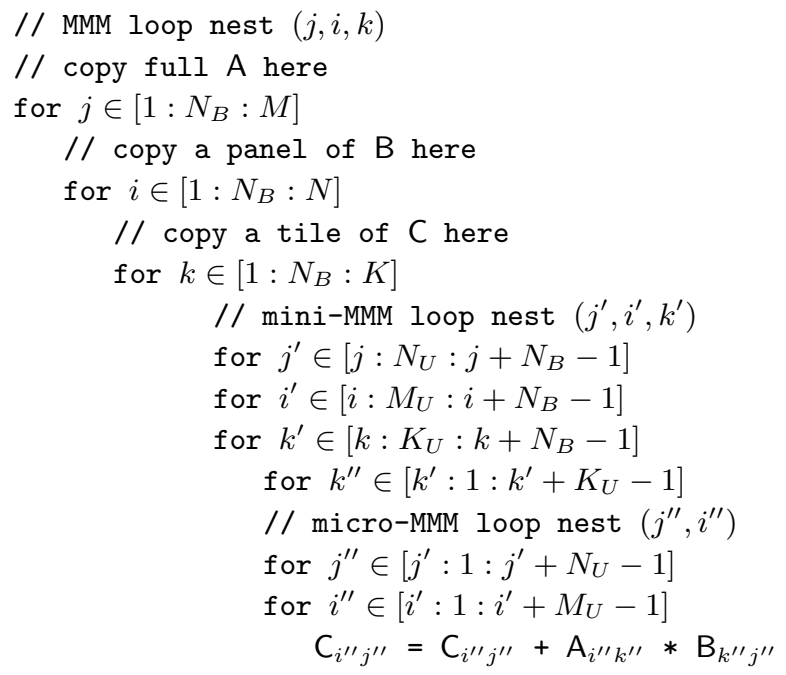

Figure 3: MMM tiled for L1 data cache and Registers

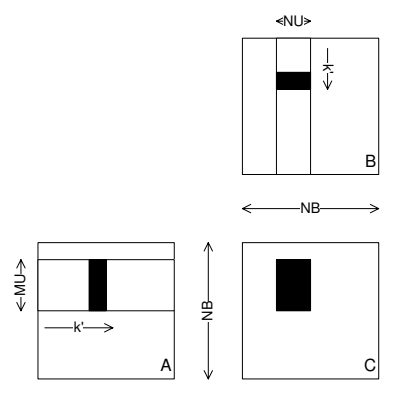

Figure 4: mini-MMM and micro-MMM

before and after the $k^{\prime}$ loop to initialize the scalars corresponding to $C$ elements, and to write the values back into the array respectively. It is expected that the back-end compiler will allocate floating-point registers for these scalars.

\subsubsection{Pipeline scheduling}

The resulting straight-line code in the body of the $k^{\prime \prime}$ loop is scheduled to make better use of the processor pipeline. Note that the operations in the $k^{\prime \prime}$ loop are the $M_{U}+N_{U}$ loads of A and B elements required for the micro-MMM, and the corresponding $M_{U} \times N_{U}$ multiplications and additions. On hardware architectures that have a fused multiply-add instruction, the scheduling problem is much simpler because multiplies and adds are executed together. Therefore, we only discuss the more interesting case when a multiply-add instruction is not present. An optimization parameter $F M A$ tells the code generator whether to assume that a fused multiply-add exists. The scheduling of operations can be described as follows.

- Construct two sequences of length $\left(M_{U} \times N_{U}\right)$, one containing the multiply operations and the other the add operations. 
- Interleave the two sequences, to create a single sequence of the form $m u l_{1} m u l_{2} \ldots m u l_{L_{s}} a d d_{1} m u l_{L_{s}+1} a d d_{2} \ldots$ that is obtained by skewing the adds by a factor of $L_{s}$, where $L_{s}$ is an optimization parameter. Intuitively, this interleaving separates most dependent multiplies and adds by $2 \times L_{s}-1$ other independent instructions to avoid stalling the processor pipeline.

- Inject the $M_{U}+N_{U}$ loads of the elements of A and B into the resulting sequence of arithmetic operations by scheduling a block of $I_{F}$ (Initial Fetch) loads in the beginning and blocks of $N_{F}$ loads thereafter as needed. $I_{F}$ and $N_{F}$ are optimization parameters.

- Unroll the $k^{\prime \prime}$ loop completely. The parameter $K_{U}$ must be chosen to be large enough to reduce loop overhead, but not so large the body of the $k^{\prime}$ loop overflows the L1 instruction cache.

- Software pipeline the $k^{\prime}$ loop in such a way that operations from the current iteration are overlapped with operations from the previous iteration.

Note that skewing of dependent adds and multiplies increases register pressure; in particular, the following inequality must hold to avoid register spills:

$$
M_{U} \times N_{U}+M_{U}+N_{U}+L_{s} \leq N_{R}
$$

\subsubsection{Discussion}

Table 1 lists the optimization parameters for future reference.

\begin{tabular}{|l||l|}
\hline Name & Description \\
\hline$N_{B}$ & L1 data cache tile size \\
$M_{U}, N_{U}$ & Register tile size \\
$K_{U}$ & Unroll factor for $k^{\prime}$ loop \\
$L_{s}$ & Latency for computation scheduling \\
$F M A$ & 1 if fused multiply-add, 0 otherwise \\
$F_{F}, I_{F}, N_{F}$ & Scheduling of loads \\
\hline
\end{tabular}

Table 1: Summary of optimization parameters

There are a few details that we have omitted. In particular, ATLAS copies tiles of $A, B$, and $C$ into sequential memory locations before performing the mini-MMM, if it thinks this would be profitable. The strategy for copying is shown in Figure 3. ATLAS also incorporates a simple form of tiling for the L2 cache, called CacheEdge; we will not discuss this because our focus in the mini-MMM code, which is independent of CacheEdge.

It is intuitively obvious that the performance of the generated mini-MMM code suffers if the values of the optimization parameters in Table 1 are too small or too large. For example, if $M_{U}$ and $N_{U}$ are too 
small, the $M_{U} \times N_{U}$ block of computation instructions might not be large enough to hide the latency of the $M_{U}+N_{U}$ loads, and performance suffers. On the other hand, if these parameters are too large, register spills will reduce performance. Similarly, if the value of $K_{U}$ is too small, there is more loop overhead, but if this value is too big, the code in the body of the $k^{\prime}$ loop will overflow the instruction cache and performance will suffer. The goal therefore is to determine optimal values of these parameters for obtaining the best mini-MMM code.

\subsection{Global search in ATLAS}

To find optimal values for the optimization parameters, ATLAS uses a global search strategy called orthogonal line search [12]. Suppose we want to find the optimal value of a function $y=f\left(x_{1}, x_{2}, \ldots x_{n}\right)$. To find an approximate solution, we reduce this $n$-dimensional optimization problem into a sequence of 1-dimensional optimization problems as follows: we order these parameters in some way and optimize them one at a time, using reference values for those that have not yet been optimized. Orthogonal line search is an approximate method in the sense that it does not necessarily find the optimal value of a function, but it might come close.

To specify an orthogonal line search, it is necessary to specify (i) the order in which the parameters are optimized, (ii) the range of possible values considered during the optimization of each parameter, and (iii) the reference value used for parameter $k$ during the optimization of parameters $1,2, \ldots, k-1$.

In ATLAS, the optimization sequence is the following: (i) $N_{B}$, (ii) $M_{U}$ and $N_{U}$, (iii) $K_{U}$, (iv) $L_{s}$ and (v) $F_{F}, I_{F}$, and $N_{F}$.

To find the best $N_{B}$, ATLAS generates a number of mini-MMMs for matrix sizes $N_{B} \times N_{B}$ where $N_{B}$ is a multiple of 4 and $16 \leq N_{B} \leq \min \left(80, \sqrt{C_{L 1}}\right)$, where $C_{L 1}$ is the capacity of the L1 data cache. During this search, the values of $M_{U}$ and $N_{U}$ are set to the values closest to each other that satisfy Inequality (1) with $M_{U}>N_{U}$. For each block size, ATLAS tries two extreme cases for $K_{U}$ no unrolling $\left(K_{U}=1\right)$ and full unrolling $\left(K_{U}=N_{B}\right)$. Suitable $L_{s}, F_{F}, I_{F}$, and $N_{F}$ are obtained from running the micro-benchmarks. The value of $N_{B}$ that produces highest MFLOPS is chosen as "best $N_{B}$ " value, and it is used from this point on in all experiments as well as in the final versions of the optimized mini-MMM code.

To find the best $M_{U}$ and $N_{U}$, ATLAS tries all combinations of $M_{U}$ and $N_{U}$ that satisfy Inequality (1). For each combination, the value of $N_{B}$ from the previous step and reference values for all other parameters are used to generate mini-MMM code; the version that performs best determines the values chosen for $M_{U}$ and $N_{U}$.

The remaining parameters are optimized in a similar way. We omit the details because they are not relevant to the rest of this paper. 


\section{Model-driven optimization}

In this section, we summarize the model of Yotov et al [19] for estimating values for optimization parameters. This model requires the following machine parameters.

- $C_{L 1}$ and $B_{L 1}$ - capacity and line size of the L1 data cache respectively

- $N_{R}$ - number of floating-point registers

- $L_{h}$ - latency in cycles of floating-point multiply

- $\left|A L U_{F P}\right|$ - number of floating-point pipes

- FMA - existence of a fused multiply-add instruction

This is a superset of the machine parameters required by the ATLAS framework. Furthermore, modelling requires more accurate values for machine parameters than searching does; models use machine parameters to directly determine optimal values for optimization parameters, whereas search-based techniques use them only to bound the search space. Therefore, our system uses a more extensive and robust set of precise micro-benchmarks [20] than ATLAS does.

\subsection{Estimating parameter values}

\subsubsection{Estimating $N_{B}$}

There has been a lot of work in the compiler community on estimating tile sizes for general programs $[3,13$, $18,9]$. In this section, we discuss a sequence of models of increasing accuracy for estimating tile sizes for matrix-multiplication.

If the three tiles of $\mathrm{A}, \mathrm{B}$, and $\mathrm{C}$ are copied into sequential memory locations, we can avoid conflict and capacity misses if all three tiles fit in the L1 cache. This leads to the following inequality.

$$
3 \times N_{B}^{2} \leq C_{L 1}
$$

This is a simple model, but on most architectures, we can afford to tolerate some misses. If we assume that we can tolerate some conflict misses but still disallow capacity misses, the tile size can be made larger since it is not necessary to keep all three tiles in the cache for the duration of the mini-MMM computation. For example, for the jik loop order, we need to keep only one element of $\mathrm{C}$ in cache, because $\mathrm{C}$ is indexed by the control variables in the outermost two loops. For this loop order, we also need to cache the complete tile of A, since we walk the full tile for each iteration of the $j$ loop. Finally we need to cache a column of 
$\mathrm{B}$, since the $j^{\text {th }}$ column is fully accessed for each iteration of the $i$ loop. Therefore, assuming that the cache has an optimal replacement policy, we obtain the following inequality:

$$
N_{B}^{2}+N_{B}+1 \leq C_{L 1}
$$

Because caches have non-unit line size, it is not possible to keep just one element of $C$ in the cache; instead we must store an entire line. Correcting for the line size $B_{L 1}$, we obtain the following inequality:

$$
\left\lceil\frac{N_{B}^{2}}{B_{L 1}}\right\rceil+\left\lceil\frac{N_{B}}{B_{L 1}}\right\rceil+1 \leq \frac{C_{L 1}}{B_{L 1}}
$$

The replacement policy in real caches is usually pseudo-LRU. Intuitively, in case of LRU replacement, we need more cache so that unwanted blocks stay there until they become least recently used. This refinement leads to the following inequality [19]:

$$
\left\lceil\frac{N_{B}^{2}}{B_{L 1}}\right\rceil+3\left\lceil\frac{N_{B}}{B_{L 1}}\right\rceil+1 \leq \frac{C_{L 1}}{B_{L 1}}
$$

Up to this point, we ignored the interaction between register tiling and L1 data cache tiling. Because of register tiling, we actually deal with vertical panels of register tiles rather than columns of scalar elements. This consideration leads to the final inequality used to compute $N_{B}$ [19].

$$
\left\lceil\frac{N_{B}^{2}}{B_{L 1}}\right\rceil+3\left\lceil\frac{N_{B} \times N_{U}}{B_{L 1}}\right\rceil+\left\lceil\frac{M_{U}}{B_{L 1}}\right\rceil \times N_{U} \leq \frac{C_{L 1}}{B_{L 1}}
$$

Once $M_{U}$ and $N_{U}$ are computed as described next, it is easy to compute $N_{B}$ from this inequality.

\subsubsection{Estimating $M_{U}$ and $N_{U}$}

Inequality (1), which is reproduced below for convenience, is used to determine $M_{U}$ and $N_{U}$. To use this inequality, we need an estimate for $L_{s}$, which is computed as described in Section 3.1.3.

$$
M_{U} \times N_{U}+M_{U}+N_{U}+L_{s} \leq N_{R}
$$

Initially, assume $M_{U}=N_{U}$. This leads to Inequality (7), which can be solved for $M_{U}$. This value can then be substituted into Inequality 1 to determine $N_{U}$.

$$
M_{U}^{2}+2 \times M_{U}+L_{s}-N_{R} \leq 0
$$


Finally, these values are adjusted to ensure that $M_{U}$ and $N_{U}$ are at least 1 and $M_{U}>N_{U}$.

\subsubsection{Estimating $L_{s}$}

From the discussion of instruction scheduling in Section 2.1, we see that a multiply operation in the innermost loop body is separated from its dependent add by a total of $2 \times\left(L_{s}-1\right)$ operations $\left(L_{s}-1\right.$ multiplies and $L_{s}-1$ adds).

The value of $L_{s}$ must be chosen so that add operations can be issued without waiting for the corresponding multiply operations to complete. If the latency of multiplication is $L_{h}$ cycles, and we have $\left|A L U_{F P}\right|$ floating

point units, it will take $\frac{2 \times\left(L_{s}-1\right)}{\left|A L U_{F P}\right|}$ cycles to issue $2 \times\left(L_{s}-1\right)$ floating point instructions. From this, Equation 8 follows.

$$
L_{h}=\frac{2 \times\left(L_{s}-1\right)}{\left|A L U_{F P}\right|}
$$

We therefore obtain the following estimate for $L_{s}$.

$$
L_{s}=\left\lceil\frac{L_{h} \times\left|A L U_{F P}\right|}{2}\right\rceil+1
$$

\subsubsection{Estimating $K_{U}$, FMA, $F_{F}, I_{F}$, and $N_{F}$}

The parameter $K_{U}$ is set so that the body of the kernel fits in the L1 instruction cache. In most cases, it is possible to completely unroll the $k^{\prime}$ loop $\left(K_{U}=N_{B}\right)$, without overflowing the L1 instruction cache. The optimization parameter FMA is set to the corresponding value measured by the hardware microbenchmarks. Finally, performance is largely insensitive to the values of the fetch parameters, so they are set to $\left(F_{F}, I_{F}, N_{F}\right)=(0,2,2)$.

\section{$3.2 \quad$ Experimental results}

We compared the performance of code generated by ATLAS and by the model-driven version of ATLAS on ten different architectures. For lack of space, in this section, we only discuss some of the more interesting performance results.

\subsubsection{AMD Opteron 240}

Table 2 shows the specifications of the AMD Opteron 240 machine we used in our experiments.

On this platform, as well as to some extent on all other x86 CISC platforms, we observed a significant performance gap between the code generated using the model and the code generated by ATLAS (see the 


\begin{tabular}{|l|r|}
\hline Feature & Value \\
\hline CPU Core Frequency & $1400 \mathrm{MHz}$ \\
L1 Data Cache & $64 \mathrm{~KB}, 64 \mathrm{~B} /$ line \\
L1 Instruction Cache & $64 \mathrm{~KB}, 64 \mathrm{~B} /$ line \\
L2 Unified Cache & $1 \mathrm{MB}, 64 \mathrm{~B} /$ line \\
Floating-Point Registers & 8 \\
Floating-Point Functional Units & 2 \\
Floating-Point Multiply Latency & 4 \\
Has Fused Multiply Add & No \\
\hline Operating System & SuSE 9 Linux \\
C Compiler & GNU GCC 3.3 \\
Fortran Compiler & GNU Fortran 3.3 \\
\hline
\end{tabular}

Table 2: AMD Opteron 240 Specifications

two lines labelled Model and Global Search in Figure 5). To understand the problem, we studied the optimization parameter values produced by the two approaches. These values are shown in Table 5 . The two sets of values are quite different, but that by itself is not necessarily significant. For example, Figure 6 shows how performance of the mini-MMM code changes as $N_{B}$ is changed, keeping all other parameters fixed. It can be seen that the values chosen by Global Search $\left(N_{B}=60\right)$ and Model $\left(N_{B}=88\right)$ are both good choices for that optimization parameter, even though they are quite different.

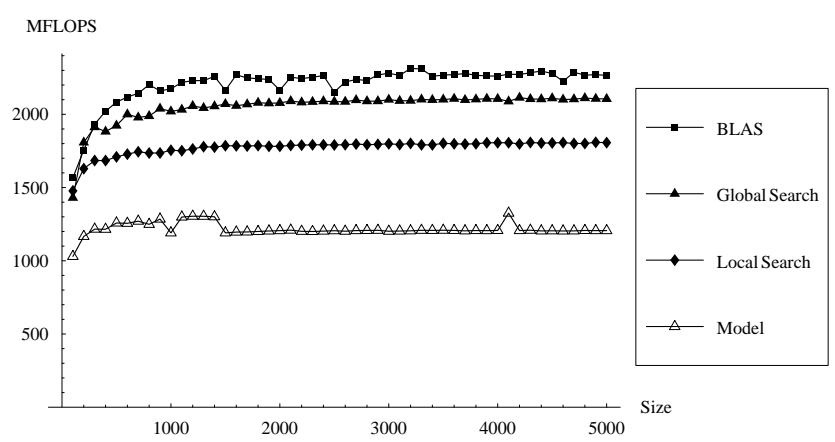

Figure 5: MMM Results for AMD Opteron 240

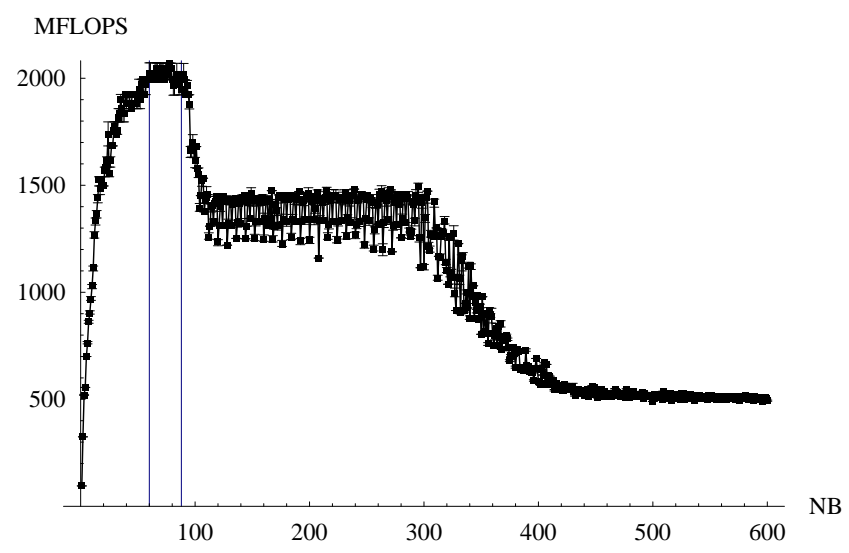

Figure 6: AMD Opteron 240 Sensitivity to $N_{B}$

On the other hand, performance sensitivity to $M_{U}$ and $N_{U}$, shown in Figure 7 , demonstrates that the 

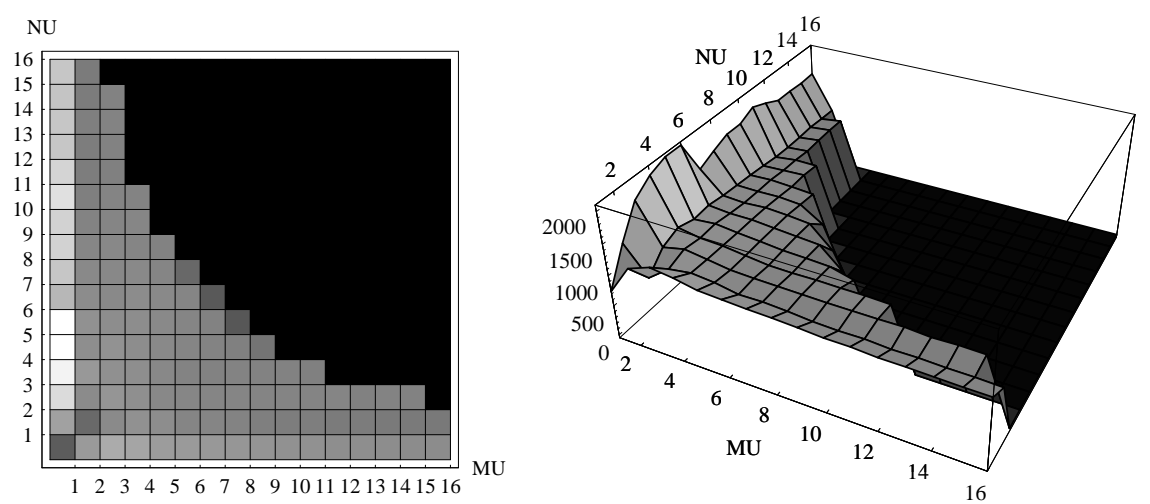

Figure 7: AMD Opteron 240 Sensitivity to $M_{U}, N_{U}$

optimal values of $\left(M_{U}, N_{U}\right)$ are $(6,1)$. Notice that this graph is not symmetric with respect to $M_{U}$ and $N_{U}$, because an $M_{U} \times 1$ tile of $\mathrm{C}$ is contiguous in memory, but a $1 \times N_{U}$ tile is not [16]. Global Search finds $\left(M_{U}, N_{U}\right)=(6,1)$, whereas the model estimates $(2,1)$. To clinch the matter, we verified that the performance difference disappears if $\left(M_{U}, N_{U}\right)$ are set to $(6,1)$, and all other optimization parameters are set to the values estimated by the model.

Since the difference in performance between the code produced by global search and the code produced by using the model is about $40 \%$, it is likely that there is a problem with the model presented in Section 3 for determining $\left(M_{U}, N_{U}\right)$. Evidence for this is provided by the line labelled Local Search in Figure 5, which shows there is significant performance gap even if we use a simple local search around the parameter values estimated by the model, which is described in more detail in Section 4.2.

In Section 4.1.1, we show how model refinement fixes this problem.

\subsubsection{SUN UltraSPARC IIIi}

\begin{tabular}{|l|r|}
\hline Feature & Value \\
\hline CPU Core Frequency & $1060 \mathrm{MHz}$ \\
L1 Data Cache & $64 \mathrm{~KB}, 32 \mathrm{~B} /$ line, 4-way \\
L1 Instruction Cache & $32 \mathrm{~KB}, 32 \mathrm{~B} /$ line, 4-way \\
L2 Unified Cache & $1 \mathrm{MB}, 32 \mathrm{~B} /$ line, 4-way \\
Floating-Point Registers & 32 \\
Floating-Point Functional Units & 2 \\
Floating-Point Multiply Latency & 4 \\
Has Fused Multiply Add & No \\
\hline Operating System & SUN Solaris 9 \\
C Compiler & SUN C 5.5 \\
Fortran Compiler & SUN FORTRAN 95 7.1 \\
\hline
\end{tabular}

Table 3: SUN UltraSPARC IIIi Specifications

Table 3 shows the specifications of the SUN UltraSPARC IIIi machine we used in our experiments.

The optimization parameters derived by using the model and global search are shown in Table 7. Figure 14 presents the MMM performance results. On this machine, Model actually performs about $10 \%$ better than 
Global Search.

However, this platform is one of several that highlights a deficiency of the model that afflicts ATLAS Global Search as well. The problem lies in the choice of $N_{B}$. Figure 8 shows the sensitivity of performance to $N_{B}$. The values chosen by Global Search, Model and the best value (44, 84 and 208 respectively) are denoted by vertical lines.

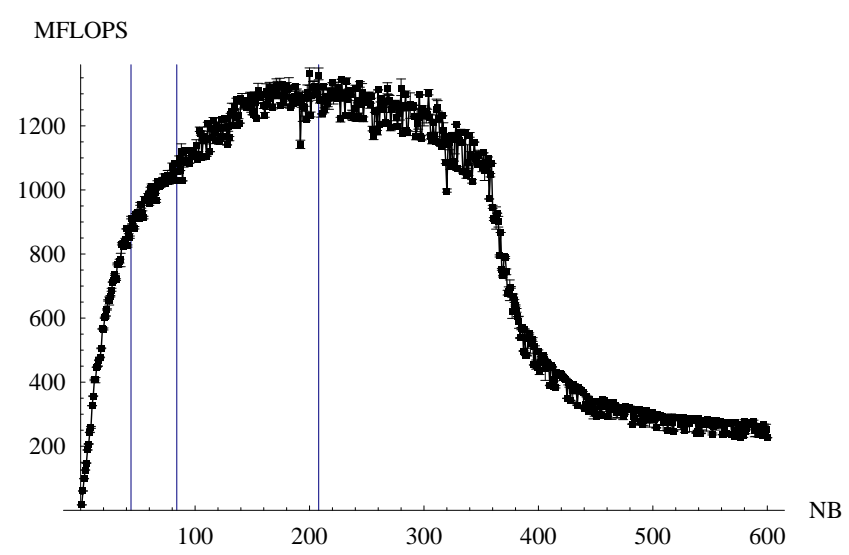

Figure 8: SUN UltraSPARC IIIi Sensitivity to $N_{B}$

Initially, performance increases with increasing values for $N_{B}$. The first, slight performance drop, at $N_{B}=208$ can be explained by applying the model in Inequality (2) but for the L2 cache $\left(C_{L 2}=1 M B\right)$. This is the point at which all three tiles fit together in the L2 cache. The second, more pronounced, performance drop, at $N_{B}=360$ can be explained by applying Inequality (6) for the L2 cache. After this point, L2 capacity misses start to occur and performance drops dramatically. Notice that there are no drops in performance around $N_{B}=52$ and $N_{B}=88$ which are the corresponding model-predicted values for the L1 data cache.

The problem is that both Model and Global Search perform tiling for the L1 cache, but it is more beneficial to tile for the L2 cache on this machine ${ }^{1}$. In general, this is desirable if (1) the cache miss latency for the L1 data cache is close to that of the L2 cache, or (2) the cache miss latency of the L1 data cache is small enough that it is possible to entirely hide almost all of the L1 data cache misses with floating point computations.

We show how model refinement solves this problem in Section 4.1.2.

\subsubsection{Intel Itanium 2}

Table 4 shows the specifications of the Intel Itanium 2 machine we used in our experiments.

\footnotetext{
${ }^{1}$ In general, another possibility is to do multi-level cache tiling but the ATLAS code generator provides support for a single level only.
} 

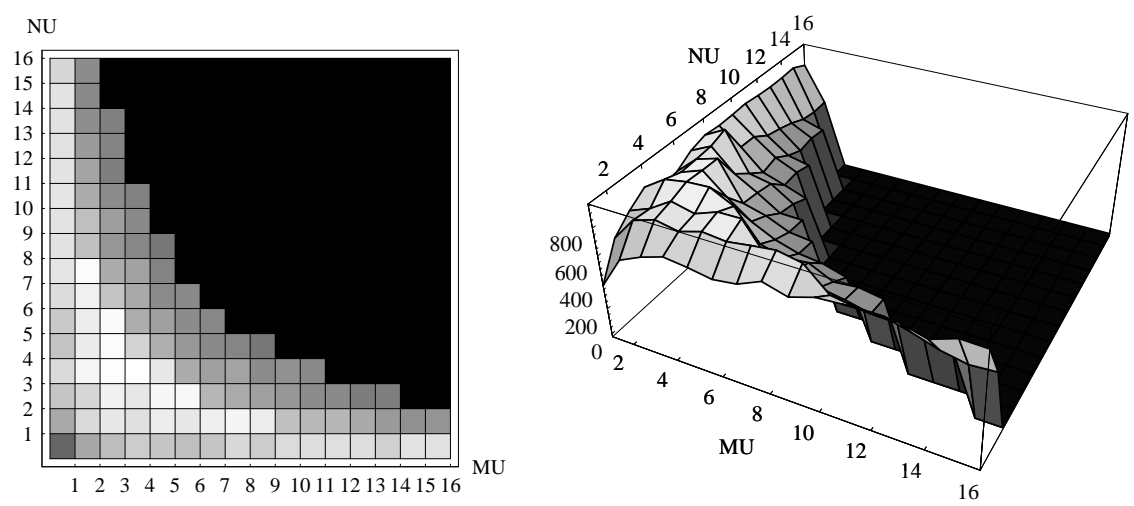

Figure 9: SUN UltraSPARC IIIi Sensitivity to $M_{U}, N_{U}$

\begin{tabular}{|l|r|}
\hline Feature & Value \\
\hline CPU Core Frequency & $1500 \mathrm{MHz}$ \\
L1 Data Cache & $16 \mathrm{~KB}, 64 \mathrm{~B} /$ line, 4-way \\
L1 Instruction Cache & $16 \mathrm{~KB}, 64 \mathrm{~B} /$ line, 4-way \\
L2 Unified Cache & $256 \mathrm{~KB}, 128 \mathrm{~B} /$ line, 8-way \\
L3 Cache & $3 \mathrm{MB}, 128 \mathrm{~B} /$ line, 12-way \\
Floating-Point Registers & 128 \\
Floating-Point Functional Units & 2 \\
Floating-Point Multiply Latency & 4 \\
Has Fused Multiply Add & Yes \\
\hline Operating System & RedHat Linux 9 \\
C Compiler & GNU GCC 3.3 \\
Fortran Compiler & GNU Fortran 3.3 \\
\hline
\end{tabular}

Table 4: Intel Itanium 2 Specifications

The values of optimization parameters estimated by using the model and global search are shown in Table 9. Figure 15 presents the MMM results.

Figure 10 shows the sensitivity of performance to $N_{B}$ on Intel Itanium 2. The values chosen by Model, Global Search, and the best value (30,80 and 360 respectively) are denoted by vertical lines.

As on the SUN UltraSPARC IIIi, tiling for the L1 data cache is not beneficial. On this platform, even the L2 cache is "invisible" to $N_{B}$ sensitivity, and the drops in performance are explained by substituting the size of the L3 cache $C_{L 3}=3 M B$ in Inequalities (2) and (6).

The second part of Figure 10 zooms into the interval $N_{B} \in[300,400]$, which contains the value that achieves best performance $\left(N_{B}=360\right)$. As we can see, there are performance spikes and dips of as much as 300 MFlops. In particular, the value of $N_{B}=362$ obtained by using Inequality (2) for the L2 cache is not nearly as good as that for $N_{B}=360$. Values of $N_{B}$ that are divisible by $M_{U}$ and $N_{U}$ usually provide slightly better performance because there are no "edge effects"; that is, no special clean-up code needs to be executed for small left-over register tiles at the boundary. The number of conflict misses in the L1 and L2 caches can also vary with tile size.

Refining the model to account for effects like conflict misses is not likely to be tractable in general, so we advocate using local search around the model-predicted value, as discussed in detail in Section 4.2.1. 

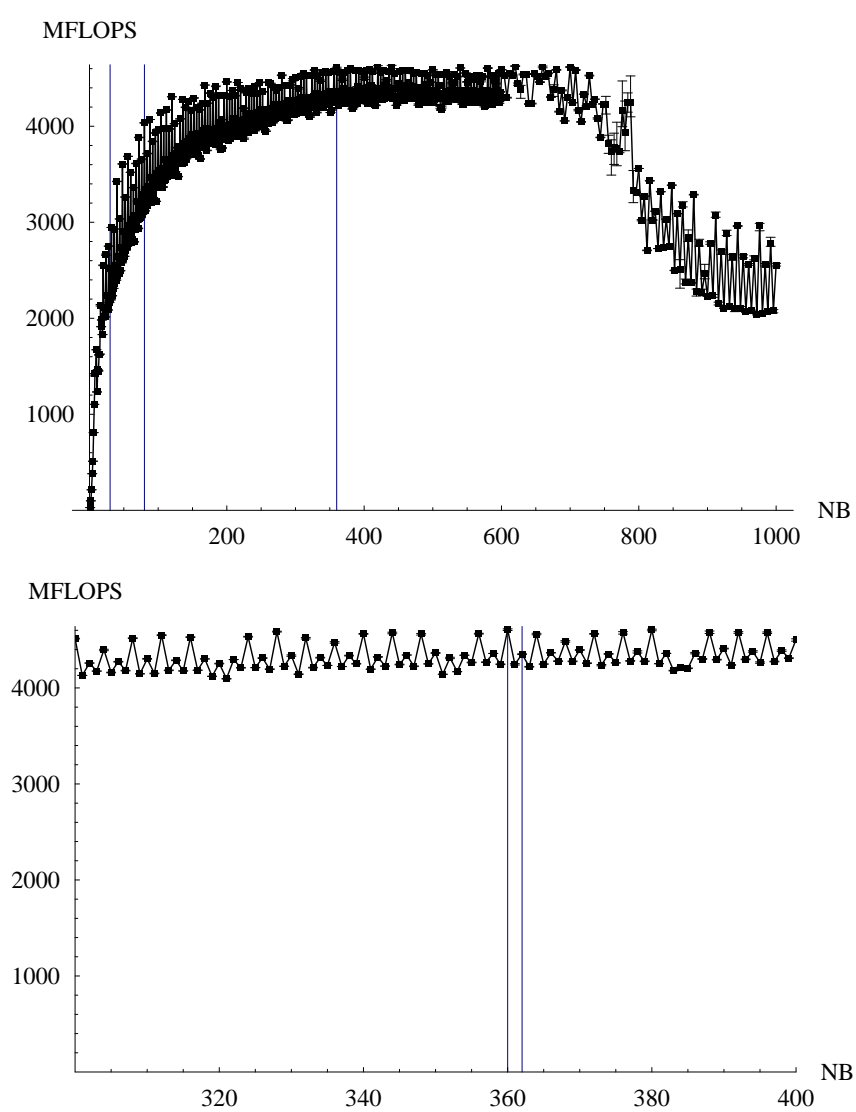

Figure 10: Intel Itanium 2 Sensitivity to $N_{B}$
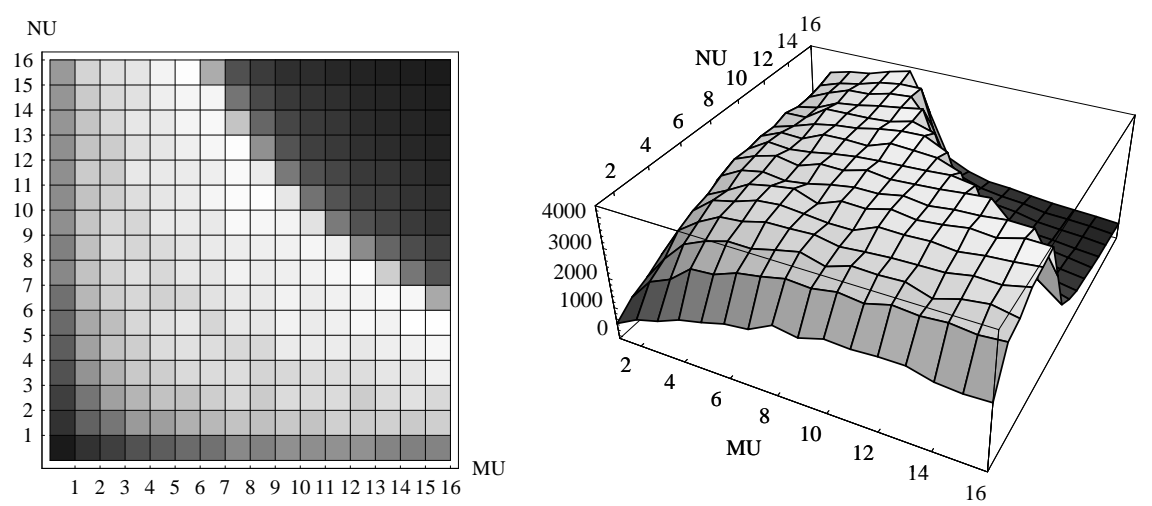

Figure 11: Intel Itanium 2 Sensitivity to $M_{U}, N_{U}$

For completeness, we show the sensitivity of performance to $M_{U}$ and $N_{U}$ in Figure 11, although there is nothing interesting in this graph. Because of the extremely large number of registers on this platform $\left(N_{R}=128\right)$, the peak of the hill is more like a plateau with a multitude of good choices for the $M_{U}$ and $N_{U}$ unroll factors. Both Model and Global Search do well. 


\subsection{Summary}

Our experiments point to two deficiencies with the model of Yotov et al [19]. On x86-based architectures like the Opteron, there is only a small number of registers, and the model does not choose $\left(M_{U}, N_{U}\right)$ optimally. On machines like the UltraSPARC IIIi and the Itanium 2, the model (and ATLAS Global Search) tile for the wrong cache level. Finally, local improvement seems useful for some parameters such as $N_{B}$ on the Itanium.

\section{Closing the gap}

We discuss model refinement in Section 4.1, and local search in Section 4.2.

\subsection{Model refinement}

\subsubsection{Choosing $M_{U}$ and $N_{U}$ for small $N_{R}$}

Recall that in Section 2, we discussed how operations within the innermost loop of the mini-MMM code are scheduled to obtain good performance even on machines without out-of-order execution. In particular, the separation between multiplies and their dependent additions increases register pressure; to avoid spills, Inequality (1), reproduced below for convenience, must hold.

$$
M_{U} \times N_{U}+M_{U}+N_{U}+L_{s} \leq N_{R}
$$

For the Opteron, Table 5 shows that the model chose $M_{U}=2, N_{U}=1, F M A=0$, while Global Search chose $M_{U}=6, N_{U}=1, F M A=1$. If instead the model had chosen, $M_{U}=6$ and $F M A=1$, while keeping the rest of the parameters the same, the mini-MMM performance rises to 2050 MFLOPS. The parameters values found by Global Search are puzzling for several reasons. First, the Opteron does not have a FMA instruction! Second, choosing 6 and 1 for the values of $M_{U}$ and $N_{U}$, violates Inequality (1) since the Opteron has only 8 registers. How can we explain this?

Recall that Inequality (1) should hold because ATLAS generates code which allocates an $M_{U} \times 1$ vectortile of matrix A (which we call $\bar{a}$ ), an $1 \times N_{U}$ vector-tile of matrix B (which we call $\bar{b}$ ) and a $M_{U} \times N_{U}$ tile of matrix $C$ (which we call $\bar{c}$ ). It then performs the outer-product of $\bar{a}$ and $\bar{b}$ and accumulates the result into $\bar{c}$. The outer-product computation requires that each element of $\bar{a}$ be multiplied by each element of $\bar{b}$, and this reuse justifies storing these vectors in registers.

Notice that if $N_{U}=1$, then $\bar{b}$ is a single scalar that is multiplied by each element of $\bar{a}$. Therefore no reuse exists for the elements of $\bar{a}$. This observation lets us generate the code in Figure 12, which uses 1 register for $\bar{b}(r b), 6$ registers for $\bar{c}\left(r c_{1} \ldots r c_{6}\right)$ and 1 temporary register $(r t)$. 


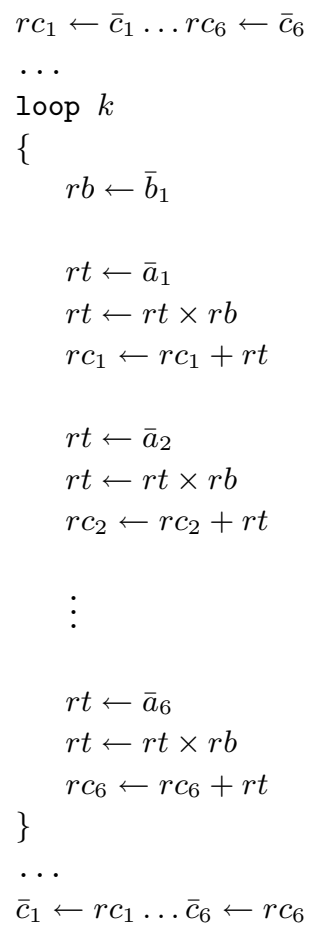

Figure 12: $\left(M_{U}, N_{U}\right)=(6,1)$ code for x86 CISC

One might expect that this code will not perform well, as there are dependences between most of the adjacent instructions because of the temporary register $r t$. In fact the code in Figure 12 performs well because of two architectural features of the Opteron.

1. Out-of-order execution: it is possible to schedule several multiplications in successive CPU cycles without waiting for their corresponding adds to complete.

2. Register renaming: the single temporary register $r t$ is renamed to a different physical register for each pair of multiply-add instructions.

Performing instruction scheduling as described in Section 2 requires additional logical registers for temporaries, which in turn limits the sizes of the register tiles. If an architecture has a small number of logical registers but the processor implements out-of-order execution and register renaming, it is better to use the logical registers for allocating larger register tiles and leave instruction scheduling to the out-of-order hardware core, which can use the extra physical registers to hold the temporaries.

These insights permit us to refine the model described in Section 3 as follows: for processors with a small number of logical registers and register renaming, set $N_{U}=1, M_{U}=N_{R}-2, F M A=1$. Section 5 describes experimental results that demonstrate that this strategy eliminates the performance gap between the code produced by model-driven optimization and the code produced by ATLAS using global search. 
Even if there are enough logical registers, this kind of scheduling may be beneficial if the ISA is 2-address rather than 3-address, because one of the operands is overwritten. This is true on the Opteron when the 16 SSE vector registers are used to hold scalar values, which is GCC's default behavior. Even though Inequality 1 prescribes $3 \times 3$ register tiles, the refined model prescribes $14 \times 1$ tiles. Experiments show that this performs better [16].

Although there is a large body of existing work on register allocation and instruction scheduling for pipelined machines $[21,6,10,15]$, we are not aware of any prior work that has highlighted this peculiar interaction between compile-time scheduling and register allocation, and dynamic register-renaming and out-of-order execution.

\subsubsection{Multilevel Memory Hierarchy}

As discussed in Section 3.2, there are some machines for which tiling for the L2 or L3 cache will give better performance than tiling for the L1 cache. The model presented in Section 3 does not account for cache miss penalties at different cache levels, so although we estimate tile sizes for different cache levels, we cannot determine which level to tile for.

One approach to addressing this problem in the context of model-driven optimization is to refine the model to include miss penalties. Our experience however is that it is difficult to use micro-benchmarks to measure miss penalties accurately for lower levels of the memory hierarchy on modern machines. Therefore, we decided to estimate tile sizes for all the cache levels according to Inequalities (2) and (6), and then empirically determine which one gives the best performance.

Notice that in the context of global search, the problem can be addressed by making the search space for $N_{B}$ large enough. However, this would increase the search time substantially since the size of an L3 cache, which would be used to bound the search space, is typically much larger than the size of an L1 cache. This difficulty highlights the advantage of our approach of using model-driven optimization together with a small amount of search - we can tackle multi-level memory hierarchies without increasing installation time significantly.

\subsection{Local search}

In this section, we describe how local search can be used to improve the $N_{B}, M_{U}, N_{U}$, and $L_{s}$ optimization parameters chosen by the model. 


\subsubsection{Local Search for $N_{B}$}

If $N_{B_{M}}$ is the value of $N_{B}$ estimated by the model, we can refine this value by local search in the interval $\left[N_{B_{M}}-\operatorname{lcm}\left(M_{U}, N_{U}\right), N_{B_{M}}+\operatorname{lcm}\left(M_{U}, N_{U}\right)\right]$. This ensures that we examine the first values of $N_{B}$ in the neighborhood of $N_{B_{M}}$ that are divisible by both $M_{U}$ and $N_{U}$.

\subsubsection{Local Search for $M_{U}, N_{U}$, and $L_{s}$}

Unlike sensitivity graphs for $N_{B}$, sensitivity graphs for $M_{U}$ and $N_{U}$ tend to be convex in the neighborhood of model-predicted values. This is probably because register allocation is under compiler control, and there are no conflict misses. Therefore, we use a simple hill-climbing search strategy to improve these parameters.

We start with the model predicted values for $M_{U}, N_{U}$, and $L_{s}$ and determine if performance improves by changing each of them by +1 and -1 . We continue following the path of increasing performance until we stop at a local maximum. On platforms on which there is a Fused-Multiply-Add instruction $(F M A=1)$, the optimization parameter $L_{s}$ has no effect on the generated code and in that case we only consider $M_{U}$ and $N_{U}$ for the hill-climbing local search.

\section{Experimental Results}

We evaluated the following six approaches on a large number of modern plarforms, including DEC Alpha 21264, IBM Power 3/4, SGI R12000, SUN UltraSPARC IIIi, Intel Pentium III/4, Intel Itanium 2, AMD Athlon MP, and AMD Opteron 240. We used ATLAS v.3.6.0, which is the latest stable version of ATLAS as of this writing.

1. Model: This approach uses the model of Yotov et al [19] as described in Section 3.

2. Refined Model: This approach uses the refined model for $\left(M_{U}, N_{U}\right)$ described in Section 4.1.1.

3. Local search: This approach uses local search as described in Section 4.2, in the neighborhood of parameter values determined by Refined Model.

4. Multi-level Local Search: This approach is the same as Local Search, but it considers tiling for lower levels of the memory hierarchy as described in Section 4.1.2.

5. Global Search: This is the ATLAS search strategy.

6. Unleashed: This is the full ATLAS distribution that includes user-contributed code, installed with accepting all defaults that the ATLAS team have provided. As such, it usually performs optimizations 
which are not exposed through the ATLAS' Code Generator and therefore it is normally not directly comparable to our results.

For lack of space, we present results only for the machines discussed earlier in this paper.

\subsection{AMD Opteron 240}

Table 5 shows the values of the optimization parameters for Model, Refined Model, Local Search, Multi-Level (ML) Local Search, Global Search, and Unleashed, along with the corresponding performance numbers for mini-MMM.

\begin{tabular}{|l||c|c|c|c|c|c|}
\hline & $N_{B}$ & $M_{U}, N_{U}, K_{U}$ & $L_{s}$ & FMA & $F_{F}, I_{F}, N_{F}$ & MFLOPS \\
\hline Model & 88 & $2,1,88$ & 2 & 0 & $0,2,2$ & 1189 \\
Refined Model & 88 & $6,1,88$ & 1 & 1 & $0,2,2$ & 2050 \\
Local Search & 88 & $6,1,88$ & 1 & 1 & $0,2,2$ & 2050 \\
ML Local Search & 88 & $6,1,88$ & 1 & 1 & $0,2,2$ & 2050 \\
Global Search & 60 & $6,1,60$ & 6 & 1 & $0,6,1$ & 2072 \\
Unleashed & 56 & & & & & 2608 \\
\hline
\end{tabular}

Table 5: Optimization Paramameters for AMD Opteron 240

Table 6 shows the times taken by our micro-benchmarks and by the ATLAS micro-benchmarks for determining machine and optimization parameters.

\begin{tabular}{|l||r|r|r|}
\hline \multicolumn{1}{|c||}{} & \multicolumn{2}{c|}{ Parameters } & Total \\
\cline { 2 - 3 } & Machine & Optimization & \\
\hline Model & 101 & 2 & 103 \\
Refined Model & 101 & 2 & 103 \\
Local Search & 101 & 31 & 132 \\
ML Local Search & 101 & 126 & 227 \\
Global Search & 148 & 375 & 523 \\
\hline
\end{tabular}

Table 6: Timings for AMD Opteron 240

Figure 13 shows the MMM performance for all approaches. Local Search and Multi-Level Local Search are not plotted on this platform, because as Table 5 suggests, their performance is virtually equivalent to that of Refined Model. For native BLAS we used ACML 2.0.

The MMM performance achieved by Model + Local Search is only marginally worse than that of Global Search, which according to our sensitivity analysis is due to a slightly suboptimal value of $N_{B}$. Had we extended the interval in which we do local $N_{B}$ search by a small amount, we would have achieved the same performance.

In summary, the model refinement described in Section 4.1.1 to take into account the small number of logical registers on this machine is sufficient to address performance problems with basic model of Yotov et al [19]. 


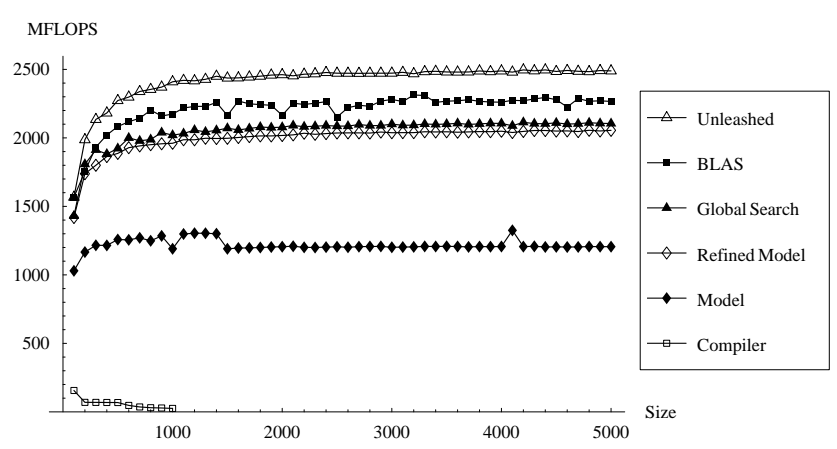

Figure 13: MMM Results for AMD Opteron 240

\section{$5.2 \quad$ SUN UltraSPARC IIIi}

Table 7 shows the values of the optimization parameters for Model, Refined Model, Local Search, Multi-Level (ML) Local Search, Global Search, and Unleashed, along with the corresponding performance numbers for mini-MMM.

\begin{tabular}{|l||c|c|c|c|c|c|}
\hline & $N_{B}$ & $M_{U}, N_{U}, K_{U}$ & $L_{s}$ & FMA & $F_{F}, I_{F}, N_{F}$ & MFLOPS \\
\hline Model & 84 & $4,4,84$ & 4 & 0 & $0,2,2$ & 1120 \\
Refined Model & 84 & $4,4,84$ & 4 & 0 & $0,2,2$ & 1120 \\
Local Search & 84 & $4,4,84$ & 4 & 0 & $0,2,2$ & 1120 \\
ML Local Search & 208 & $4,4,16$ & 4 & 0 & $0,2,2$ & 1308 \\
Global Search & 44 & $4,3,44$ & 5 & 0 & $0,3,2$ & 986 \\
Unleashed & 168 & & & & & 1694 \\
\hline
\end{tabular}

Table 7: Optimization Paramameters for SUN UltraSPARC IIIi

Table 8 shows the times taken by our micro-benchmarks and by the ATLAS micro-benchmarks for determining machine and optimization parameters.

\begin{tabular}{|l||r|r|r|}
\hline \multicolumn{1}{|c||}{} & \multicolumn{2}{c|}{ Parameters } & Total \\
\cline { 2 - 3 } & Machine & Optimization & (sec) \\
\hline Model & 112 & 7 & 119 \\
Refined Model & 112 & 7 & 119 \\
Local Search & 112 & 127 & 239 \\
ML Local Search & 112 & 496 & 608 \\
Global Search & 203 & 1233 & 1436 \\
\hline
\end{tabular}

Table 8: Timings for SUN UltraSPARC IIIi

Figure 14 shows the MMM performance for all approaches. Refined Model and Local Search are not plotted on this platform, because as Table 7 suggests, their performance is virtually equivalent to that of Model. We used the native BLAS library included in Sun One Studio 9.0.

Model performs marginally better than Global Search because the ATLAS micro-benchmarks estimated that the $\mathrm{L} 1$ data cache size is $16 \mathrm{~KB}$, rather than $64 \mathrm{~KB}$. This overly restricted the $N_{B}$ interval examined by Global Search, leading to poor performance. Multi-Level Local Search performs better than Local Search because it finds that it is better to tile for the L2 cache rather than for the L1. 


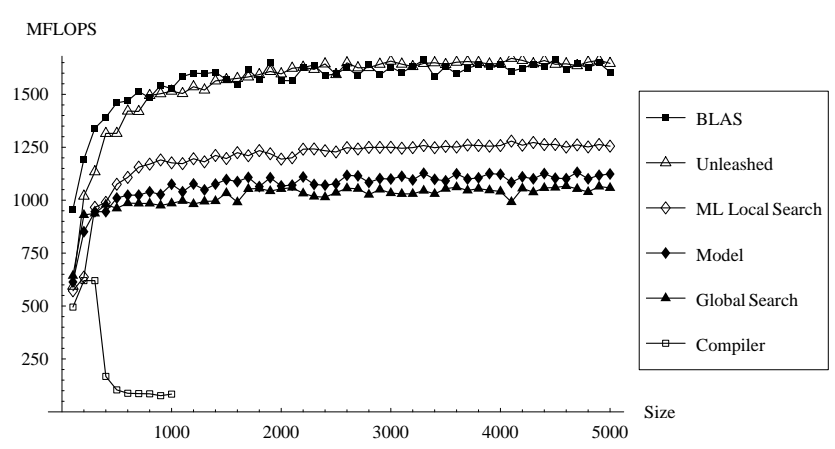

Figure 14: MMM Results for SUN UltraSPARC IIIi

\subsection{Intel Itanium 2}

The description of this platform was presented in Table 4 .

Table 9 shows the values of the optimization parameters for Model, Refined Model, Local Search, MultiLevel (ML) Local Search, Global Search, and Unleashed, along with the corresponding performance numbers for mini-MMM.

\begin{tabular}{|l||c|c|c|c|c|c|}
\hline & $N_{B}$ & $M_{U}, N_{U}, K_{U}$ & $L_{s}$ & FMA & $F_{F}, I_{F}, N_{F}$ & MFLOPS \\
\hline Model & 30 & $10,10,4$ & 1 & 1 & $0,2,2$ & 3130 \\
Refined Model & 30 & $10,10,4$ & 1 & 1 & $0,2,2$ & 3130 \\
Local Search & 30 & $10,10,4$ & 1 & 1 & $0,2,2$ & 3130 \\
ML Local Search & 360 & $10,10,4$ & 1 & 1 & $0,2,2$ & 4602 \\
Global Search & 80 & $10,10,4$ & 4 & 1 & $0,19,1$ & 4027 \\
Unleashed & 120 & & & & & 4890 \\
\hline
\end{tabular}

Table 9: Optimization Paramameters for Intel Itanium 2

Table 10 shows the times taken by our micro-benchmarks and by the ATLAS micro-benchmarks for determining machine and optimization parameters.

\begin{tabular}{|l||r|r|r|}
\hline \multicolumn{1}{|c||}{} & \multicolumn{2}{c|}{ Parameters } & Total \\
& Machine & Optimization & $(\mathrm{sec})$ \\
\hline Model & 143 & 6 & 149 \\
Refined Model & 143 & 6 & 149 \\
Local Search & 143 & 162 & 305 \\
ML Local Search & 143 & 278 & 421 \\
Global Search & 1554 & 29667 & 31221 \\
\hline
\end{tabular}

Table 10: Timings for Intel Itanium 2

Figure 15 shows the MMM performance for all these approaches. Refined Model and Local Search are not plotted on this platform, because their performance is virtually equivalent to that of Model. Native BLAS used is MKL 6.1.

Model does not perform well because it tiles for the L1 data cache. For the Itanium, ATLAS used the size of the L2 cache $(256 \mathrm{~KB})$ to restrict $N_{B}$, effectively selecting the maximum value in the search range $\left(N_{B}=80\right)$. Nevertheless this tile size is not optimal either. The Multi-level model determined that tiling for the 3 MB L3 cache is optimal, and chooses a value of $N_{B}=362$. This is refined to $N_{B}=360$ by local 


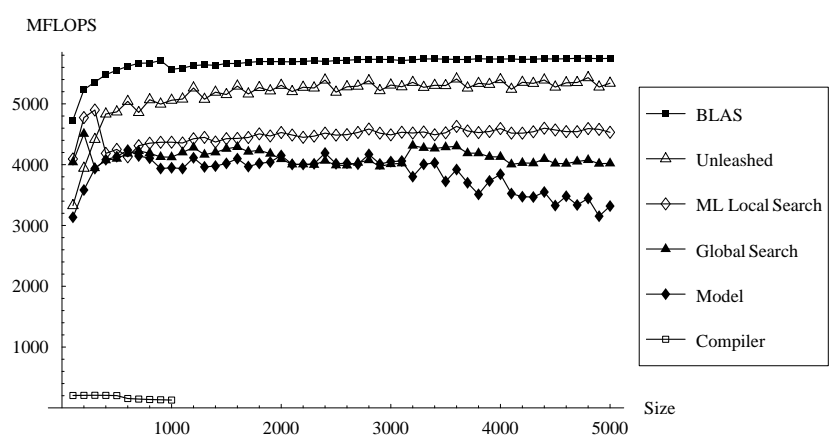

Figure 15: MMM Results for Intel Itanium 2

search. This improves performance compared to both Model and Global Search.

\subsection{SGI R12000}

\begin{tabular}{|l|r|}
\hline Feature & Value \\
\hline CPU Core Frequency & $270 \mathrm{MHz}$ \\
L1 Data Cache & $32 \mathrm{~KB}, 32 \mathrm{~B} /$ line, 2-way \\
L1 Instruction Cache & $32 \mathrm{~KB}, 32 \mathrm{~B} /$ line, 2-way \\
L2 Unified Cache & $4 \mathrm{MB}, 32 \mathrm{~B} /$ line, 1-way \\
Floating-Point Registers & 32 \\
Floating-Point Functional Units & 2 \\
Floating-Point Multiply Latency & 2 \\
Has Fused Multiply Add & No \\
\hline Operating System & IRIX64 \\
C Compiler & SGI MIPSPro C 7.3.1.1m \\
Fortran Compiler & SGI MIPSPro FORTRAN 7.3.1.1m \\
\hline
\end{tabular}

Table 11: SGI R12000 Specifications

Table 11 shows the specifications of the SGI R12000 machine we used in our experiments.

Table 12 shows the values of the optimization parameters for Model, Refined Model, Local Search, MultiLevel (ML) Local Search, Global Search, and Unleashed, along with the corresponding performance numbers for mini-MMM.

\begin{tabular}{|l||c|c|c|c|c|c|}
\hline & $N_{B}$ & $M_{U}, N_{U}, K_{U}$ & $L_{s}$ & FMA & $F_{F}, I_{F}, N_{F}$ & MFLOPS \\
\hline Model & 58 & $5,4,58$ & 1 & 1 & $0,2,2$ & 440 \\
Refined Model & 58 & $5,4,58$ & 1 & 1 & $0,2,2$ & 440 \\
Local Search & 58 & $5,4,58$ & 1 & 1 & $0,2,2$ & 440 \\
ML Local Search & 418 & $5,4,16$ & 1 & 1 & $0,2,2$ & 508 \\
Global Search & 64 & $4,5,64$ & 1 & 0 & $1,8,1$ & 457 \\
Unleashed & 64 & & & & & 463 \\
\hline
\end{tabular}

Table 12: Optimization Paramameters for SGI R12000

Table 13 shows the times taken by our micro-benchmarks and by the ATLAS micro-benchmarks for determining machine and optimization parameters.

Figure 16 shows the MMM performance on the SGI R12K. Refined Model and Local Search are not plotted on this platform, because as Table 12 suggests, their performance is virtually equivalent to that of Model. For native BLAS we used SGI SCSL v.1.4.1.3. 


\begin{tabular}{|l||r|r|r|}
\hline \multicolumn{1}{|c||}{} & \multicolumn{2}{c|}{ Parameters } & Total \\
\cline { 2 - 3 } & Machine & Optimization & (sec) \\
\hline Model & 118 & 13 & 131 \\
Refined Model & 118 & 13 & 131 \\
Local Search & 118 & 457 & 575 \\
ML Local Search & 118 & 496 & 608 \\
Global Search & 251 & 2131 & 2382 \\
\hline
\end{tabular}

Table 13: Timings for SGI R12000

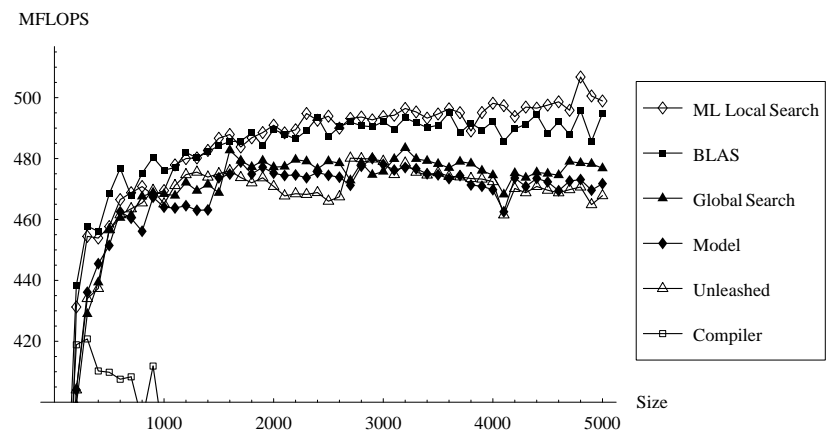

Figure 16: MMM Results for SGI R12000

The most interesting fact on this platform is that Multi-Level Local Search successfully finds that it is worth tiling for the L2 cache. By doing this, it achieves better performance than even the native BLAS. Global Search achieves slightly better performance than Model due to the minor differences in several optimization parameters. Unleashed does fine for relatively small matrices but for large ones performs worse than Model and Global Search. Although not entirely visible from the plot, on this platform, the native compiler (SGI MIPSPro) does a relatively good job.

\section{Conclusions and Future Work}

The compiler community has invested considerable effort in inventing program optimization strategies which can produce high-quality code from high-level programs, and which can scale to large programs and complex architectures. In spite of this, current compilers produce very poor code even for a simple kernel like matrix multiplication. To make progress in this area, we believe it is necessary to perform detailed case studies.

This paper reports the results of one such case study. Previously, Yotov et al [19] have shown that modeldriven optimization can produce BLAS codes with performance within 10-20\% of that of code produced by empirical optimization. We have shown that this remaining performance gap can be eliminated by a combination of model refinement and local search, without increasing search time substantially. The model refinement (i) corrects the instruction scheduling strategy for machines on which there are relatively few logical registers, and (ii) opens up the possibility of tiling for lower levels of the memory hierarchy. On some machines, this gave better performance than both ATLAS Global Search and the native BLAS. 
We believe that this combination of model refinement and local search is promising, and it is the cornerstone of a system we are building for generating dense numerical linear algebra libraries that are optimized for many levels of the memory hierarchy, a problem for which global search is not tractable. We also believe that this approach is the most promising one for incorporation into general-purpose compilers.

\section{References}

[1] B. Calder, K. Chandra, S. John, and T. Austin. Cache-conscious data placement. In Proceedings of the Eighth International Conference on Architectural Support for Programming Languages and Operating Systems (ASPLOS-VIII), San Jose, 1998.

[2] Michal Cierniak and Wei Li. Unifying data and control transformations for distributed shared memory machines. In SIGPLAN Conference on Programming Language Design and Implementation, pages 205$217,1995$.

[3] Stephanie Coleman and Kathryn S. McKinley. Tile size selection using cache organization and data layout. In SIGPLAN Conference on Programming Language Design and Implementation, pages 279-290, 1995.

[4] Keith D. Cooper, Philip J. Schielke, and Devika Subramanian. Optimizing for reduced code space using genetic algorithms. In Proceedings of the ACM SIGPLAN 1999 workshop on Languages, compilers, and tools for embedded systems, pages 1-9. ACM Press, 1999.

[5] Jack Dongarra. Personal communication.

[6] Evelyn Duesterwald, Rajiv Gupta, and Mary Lou Soffa. Register pipelining: An integrated approach to register allocation for scalar and subscripted variables. In Proceedings of the 4 th International Conference on Compiler Construction, pages 192-206. Springer-Verlag, 1992.

[7] J. L. Hennessy and D. A. Patterson. Computer Architecture: A Quantitative Approach. Morgan Kaufmann Publishers, 1990.

[8] Mark Horowitz, Margaret Martonosi, Todd C. Mowry, and Michael D. Smith. Informing memory operations: Memory performance feedback mechanisms and their applications. ACM Transactions on Computer Systems, 16(2):170-205, May 1998. 
[9] Monica D. Lam, Edward E. Rothberg, and Michael E. Wolf. The cache performance and optimizations of blocked algorithms. In Proceedings of the fourth international conference on Architectural support for programming languages and operating systems, pages 63-74. ACM Press, 1991.

[10] Daniel M. Lavery, Pohua P. Chang, Scott A. Mahlke, William Y. Chen, and Wen mei W. Hwu. The importance of prepass code scheduling for superscalar and superpipelined processors. IEEE Trans. Comput., 44(3):353-370, 1995.

[11] Sungdo Moon, Byoungro So, Mary W. Hall, and Brian R. Murphy. A case for combining compile-time and run-time parallelization. In Selected Papers from the 4th International Workshop on Languages, Compilers, and Run-Time Systems for Scalable Computers, pages 91-106. Springer-Verlag, 1998.

[12] William Press, Saul Teukolsky, William Vetterling, and Brian Flannery. Numerical Recipes in C. Cambridge University Press, 2002.

[13] Robert Schreiber and Jack Dongarra. Automatic blocking of nested loops. Technical Report CS-90-108, Knoxville, TN 37996, USA, 1990.

[14] Xipeng Shen, Yutao Zhong, and Chen Ding. Locality phase prediction. In Proceedings of Eleventh International Conference on Architectural Support for Programming Languages and Operating Systems (ASPLOS XI), 2004.

[15] Eric Sprangle and Yale Patt. Facilitating superscalar processing via a combined static/dynamic register renaming scheme. In Proceedings of the 27th annual international symposium on Microarchitecture, pages 143-147. ACM Press, 1994.

[16] R. Clint Whaley. http://sourceforge.net/mailarchive/forum.php?thread_id=1569256\&forum_ $i d=426$.

[17] R. Clint Whaley, Antoine Petitet, and Jack J. Dongarra. Automated Empirical Optimization of Software and the ATLAS Project. http://www.netlib.org/lapack/lawns/lawn147.ps.

[18] Michael E. Wolf, Dror E. Maydan, and Ding-Kai Chen. Combining loop transformations considering caches and scheduling. In Proceedings of the 29th annual ACM/IEEE international symposium on Microarchitecture, pages 274-286. IEEE Computer Society, 1996.

[19] Kamen Yotov, Xiaoming Li, Gang Ren, Michael Cibulskis, Gerald DeJong, Maria Garzaran, David Padua, Keshav Pingali, Paul Stodghill, and Peng Wu. A comparison of empirical and model-driven 
optimization. In Proceedings of the ACM SIGPLAN 2003 conference on Programming language design and implementation, pages 63-76. ACM Press, 2003.

[20] Kamen Yotov, Keshav Pingali, and Paul Stodghill. X-Ray: Automatic measurement of hardware parameters. Technical Report TR2004-1966, October 2004.

[21] Huiyang Zhou and Thomas M. Conte. Code size efficiency in global scheduling for ILP processors. In Sixth Annual Workshop on Interaction between Compilers and Computer Architectures, February 2002. 